However, the Parc National des Volcans comprises less than a third of the gorillas' total range in the Virunga Volcanoes, and the animals cannot survive there unless they are also adequately protected in the other two countries involved, Uganda and Zaire. The next step, therefore, must be to strengthen the protection in these two countries, perhaps by a similar injection of funds and expertise, and, very importantly, to encourage implementation of recommendations already drawn up during tripartite meetings on co-operation in the whole Virunga conservation area. At the moment the countries act independently of each other; they need to work together.

A. H. HARCOURT

\title{
Mountain Gorilla Fund
}

With a target of $£ 50,000$ the Mountain Gorilla Fund had raised $£ 24,325$ by February 15. FPS is exceedingly grateful to all who have contributed. The following sums of $£ 20$ and over were received before February 15:

People's Trust for Endangered Species

R. Kuok

Friends of Howletts and Port Lympne

Sarah Jackson Trust, Dr E. G. Tutin, RSPCA Overseas Fund,

R. Doyle, each

P. Panaman

$£ 13,000$

$£ 500$

$£ 300$

Mrs N. Fortuin, Cambos Enterprises Ltd, G. C. K. Dunsterville,

T. Golightly, H. A. E. Baker, T. Boyer, each

A. H. \& K. S. Harcourt

Miss A. S. Albertson

V. A. Rudderham, Dr K. Lang, L. Snelling, S. J. N. Newbound,

$£ 100$

$\$ 70$

$£ 62$

W. Foster, Mrs C. Sheridan-Johnson, B. J. Margaretts, D. W. M. Easton,

S. E. H. Bicester, Mrs P. J. Wakeman, F. A. Chamberlain, each

D. J. Cole, P. J. Bloomfield, each

R. Whittingham

D. Scharf, Mrs S. A. M. Heathcote-Amory, Mr \& Mrs P. Evans, each

P. Burn, Bruce Coleman, Margaret Davey, Ned Davey, E. A. L. Cole,

R. Harcourt, Mrs J. Reid, P. Carder, M. Simons, Mrs R. Williams,

D. Fisher, each

K. Richards, C. A. Moody, F. Crowther, G. L. Bradshaw,

R. G. Marshall-Andrews, C. Frater, Mrs M. F. Singleton,

Dr M. K. Hudson, M. Bickerstaff, Dr J. Augstein, M. J. Noble,

D. Jones, S. Low, A. Marshall, J. M. D. \& M. B. Couchman,

Mrs B. A. New, P. J. Andrade, Mrs P. M. Jenkins, E. A. \&

E. K. Wicks, S. A. Harcourt, each

\section{New FPS Overseas Consultants}

\author{
Dr Kim Hon Kuy \\ Dr Brydget Hudson \\ $\mathrm{Mr}$ Noritake Ichida \\ Mr Faisal A. Izzeddin \\ Mr. James S. Laird \\ Dr C. McDougal \\ Mr Hemanta Mishra
}

South Korea
Papua New Guinea
Japan
Bahrain
Kenya
Nepal
Nepal

FPS now has 111 consultants in 69 countries 